\title{
Correction to: Low Seroprevalence of Neutralizing Antibodies Targeting Two Clade F AAV in Humans, by Ellsworth JL, O'Callaghan M, Rubin $H$, and Seymour A. Hum Gene Ther Clin Devel 2017;29(1):60-67. DOI: 10.1089/humc.2017.239
}

Tn the March 2018 issue of Human Gene Therapy Clinical Development (vol. 29, no. 1, pages 60-67), in 1 the article entitled, Low Seroprevalence of Neutralizing Antibodies Targeting Two Clade F AAV in Humans by J.L. Ellsworth et al., there was an error in the Materials and Methods section, under NAb analyses (page 61).

The following sentence was written as:

Since mouse serum in the presence of AAV activates $\beta$-galactosidase activity in the Huh7 cell assay in a dose-dependent manner (up to 15 - to 2 -fold at a $1 / 5$ serum dilution), a $50 \%$ inhibition of cellular transduction at a 1/16 dilution of serum was used as cutoff for seropositivity for each AAV to minimize the number of false-positives.

It should have read:

Since mouse serum in the presence of AAV activates $\beta$-galactosidase activity in the Huh7 cell assay in a dose-dependent manner (up to 15 - to 20 -fold at a $1 / 5$ serum dilution), a $50 \%$ inhibition of cellular transduction at a 1/16 dilution of serum was used as cutoff for seropositivity for each AAV to minimize the number of false-positives.

The online version of the article has been corrected to reflect this change. The authors wish to apologize for the error. 